\title{
Usando dados ligados na representação de indicadores da inovação regional
}

\author{
Rafael de Moura Speroni \\ Evelin Priscila Trindade \\ Doutor em Engenharia e Gestão do Conhecimento, Instituto Federal Catarinense (IFC), Brasil - rafael@araquari.ifc.edu.br \\ Mestre em Engenharia e Gestão do Conhecimento, Universidade Federal de Santa Catarina (UFSC), Brasil - \\ evelin.trindade@gmail.com \\ Marcelo Macedo Doutor em Engenharia e Gestão do Conhecimento, Universidade Federal de Santa Catarina (UFSC) - Brasil - \\ marcelomacedo@egc.ufsc.br \\ Fernando Alvaro 0stuni Gauthier \\ Rogério Cid Bastos \\ Doutor em Engenharia de Produção, Universidade Federal de Santa Catarina (UFSC), fernando.gauthier@gmail.com \\ Doutor em Engenharia de Produção, Universidade Federal de Santa Catarina (UFSC), rogerio.bastos@ufsc.br
}

\begin{abstract}
Resumo
Quando considerado sob um contexto regional, o complexo sistema que caracteriza o processo de inovação recebe o nome de Sistema Regional de Inovação, representando um ambiente heterogêneo, onde diferentes tipos de atores interagem e coevoluem. Dada a sua complexidade, diferentes índices são apresentados na literatura para a mensuração da inovação ao nível das empresas ou em nível nacional, mas que são de difícil aplicação em nível regional, em função da subjetividade de escolha das variáveis e da falta de disponibilidade de dados. Este artigo apresenta um modelo de referência concebido a partir da análise dos modelos de indicadores compostos para mensuração da inovação regional apresentados na literatura, propondo uma classificação e representação da conceituação para os indicadores de inovação regional. O modelo é suportado por dados ligados, objetivando a exploração do potencial de dados regionais disponibilizados na Web por iniciativas de dados abertos e transparência pública na definição de índices específicos para a inovação regional.
\end{abstract}

Palavras-chave: inovação; indicadores de inovação; inovação regional; dados ligados abertos.

\section{Using linked data to represent regional innovation indicators}

\begin{abstract}
Innovation is characterized by a complex system where different kinds of players interact and coevolve in a heterogeneous environment. When considered in a regional level those systems are called Regional Innovation Systems. Due to its complexity, many different kinds of indicators are presented in the literature to measure innovation at company level or at the national level, but they are difficult to implement in a regional level, because of the subjectivity of the choice of variables and the lack of data available. This paper proposes a reference model designed based on the analysis of indicators of models for measurement of regional innovation in the literature by proposing a classification and representation of the regional innovation indicators. The model is supported by linked data, aiming at exploring the potential regional data available on the Web for open data initiatives and public transparency in the definition of specific indexes for regional innovation.
\end{abstract}

Keywords: innovation; innovation indicators; regional innovation; open linked data. 


\section{INTRODUÇÃO}

Com base em teorias econômicas, passou-se a analisar a inovação por seu papel na economia de regiões como forma de explicar a organização das atividades econômicas no espaço, bem como o crescimento ou estagnação destas ao longo do tempo. O argumento é de que o conjunto de atores da região produz efeitos sistêmicos que encorajam as empresas a desenvolver formas específicas de capital que derivam das relações sociais, normas, valores e interações com a comunidade, reforçando a competitividade e a capacidade de inovação regional (Doloreaux \& Parto, 2004).

Nos últimos anos, os governos de vários países, incluindo o Brasil, vêm demonstrando crescente preocupação com a necessidade de participação da sociedade no governo. Dentre as iniciativas neste sentido, salienta-se a publicação de dados abertos por órgãos governamentais. A motivação pela disponibilização de dados é justificada pela esperança do aumento do envolvimento dos cidadãos no governo, do aumento da transparência e da melhoria na tomada de decisão (Bertot \& Choi, 2013; Conradie \& Choenni, 2014).

Este artigo apresenta resultados de uma pesquisa realizada para uma tese de doutorado na área de engenharia do conhecimento. A abordagem proposta tem por objetivo criar um modelo de referência para indicadores de inovação regional. Embasado na literatura sobre inovação, no conceito de sistemas regionais de inovação e em modelos de índices de inovação regional coletados na literatura, o modelo de referência deverá servir de base para a definição de modelos específicos de índices de inovação regional suportados por Dados Ligados.

As seções deste estudo serão apresentadas conforme a sequência: inovação, indicadores de inovação, dados ligados abertos, metodologia e considerações finais.

\subsection{Objetivo geral}

Apresentar uma proposta de modelo de referência para representação de indicadores da inovação regional suportado por dados ligados.

\subsection{Objetivos específicos}

- Caracterizar os indicadores da inovação regional;

- Mostrar uma classificação para os indicadores da inovação regional;

- Aplicar as tecnologias de dados ligados para criação de um modelo de referência;

- Apresentar um processo de publicação de dados ligados referenciando o modelo proposto.

\section{INOVAÇÃO}

O termo "inovação" é notoriamente ambíguo e falta uma definição ou medida única (Adams, Bessant, \& Phelps, 2006). Para Schumpeter (2012), a inovação pode assumir várias formas, não sendo necessário que se invente algo novo, podendo mesmo submeter uma ideia já existente a uma nova forma de realizá-la ou uma nova situação. Mais especificamente, Crossan and Apaydin (2010, p. 02) definem:

"Inovação é: produção ou adoção, assimilação e exploração de novidades de valor-agregado em esferas econômicas e sociais; renovação e ampliação de produtos, serviços e mercados; desenvolvimento de novos métodos de produção; e estabelecimento de novos sistemas de gestão. É tanto o processo quanto o resultado".

No Manual de Oslo (OECD, 2005), referência para a padronização de conceitos, metodologias e construção de estatísticas e indicadores de Pesquisa e Desenvolvimento (P\&D), define-se: "Uma inovação é a implementação de um novo, ou significativamente melhorado, produto (bem ou serviço), ou processo, um novo método de marketing, ou novo método organizacional em práticas de negócio, organização do ambiente de trabalho, ou relações externas" (OECD, 2005, p. 46).

Inovação pode significar uma nova e única aplicação de antigas tecnologias, usando projetos para o desenvolvimento de novos produtos e serviços, novos processos e estruturas para melhorar o desempenho em diversas áreas. Em função deste entendimento, a inovação hoje está, cada vez mais, indo além dos limites da P\&D formal para redefinir tudo (National Inovation Council, 2010). Para interpretar e medir os resultados da inovação, são utilizados os indicadores de inovação. 


\subsection{Indicadores de Inovação}

Indicadores de inovação são atividades desenvolvidas por diversas entidades, que coletam e interpretam dados sobre a inovação e divulgam seus resultados na forma de publicações com periodicidades diferentes, com o objetivo de possibilitar a avaliação das atividades de inovação desenvolvidas em determinados contextos. Comumente, deseja-se estabelecer comparações entre países, sendo definidos índices de inovação que agregam conjuntos de indicadores (Cornell University, INSEAD, \& WIPO, 2013; European Union, 2014).

Em sua concepção, tais publicações baseiam-se nas teorias de inovação e desenvolvem modelos de indicadores compostos, cujos dados são coletados por meio da realização de surveys específicos que seguem orientações de guias de coleta e interpretação de dados da inovação, como os manuais de Oslo (OECD, 2005) e Frascati (OECD, 2015). Em outros casos, como no Global Innovation Index, no European Innovation Scoreboard e Regional Innovation Scoreboard, são desenvolvidos modelos que utilizam como insumos os indicadores produzidos por publicações de terceiros, como as do Banco Mundial, OECD, FMI (Cornell University et al., 2013; European Union, 2014).

\section{DADOS LIGADOS ABERTOS}

O termo Dados Ligados (Linked Data), proposto por Berners-Lee (2006), refere-se a um estilo de publicar e interligar dados estruturados de diferentes fontes na Web. O assunto desperta considerável interesse acadêmico e diversas ações estão sendo desenvolvidas no sentido da criação de repositórios de dados ligados (Bizer, Heath, \& BernersLee, 2009; Bradley, 2009; Heath, 2011). O objetivo é a construção de um espaço global de dados baseado em padrões abertos, a Web de Dados, cuja proposta é ser uma extensão da Web atual, através de um paradigma de publicação, não apenas documentos, mas também de dados (Heath, 2011).

Para que seja possível atingir o objetivo da Linked Data, de forma que os dados passem a fazer parte de um repositório global, Berners-Lee (2006) apresenta quatro regras, conhecidas como os princípios da Linked Data:

1) Deve-se usar URIs (Uniform Resource Identifiers) como nomes para as coisas;

2) Utilizar HTTP URIs (Hyper Text Transfer Protocol) de modo que as pessoas possam procurar por esses nomes;

3) Quando alguém procurar um URI, fornecer informações úteis usando os padrões RDF (Resource Description Framework) e SPARQL (linguagem de consulta); e

4) Incluir links para outros URIs, a fim de que se possa descobrir mais coisas.

As práticas de Linked Data oferecem um mecanismo simples para combinação de múltiplas fontes na Web (Hyland \& Wood, 2011), fornecendo uma gama de padrões internacionais e melhores práticas para a publicação, divulgação e reutilização de dados estruturados.

Caracterizam-se os Dados Abertos Ligados (Linked Open Data - LOD) quando os dados ligados são publicados segundo os critérios que os caracterizam como dados abertos, definidos pela Open Definition (2009) como aqueles que qualquer pessoa pode livremente usar, reutilizar e redistribuir, estando sujeito, no máximo, à exigência de creditar a sua autoria e compartilhá-los segundo a mesma licença.

Há muitos usos internos importantes para dados ligados, e para uso em dados pessoais e de grupos (Berners-Lee, 2006). Assim como muitos dados abertos não são dados ligados, os dados publicados na forma de Linked Data não precisam, necessariamente, ser abertos.

Governos, como os do Canadá, Estados Unidos, Reino Unido, França, Espanha, Suécia, Itália, Hong Kong, Austrália, Nova Zelândia e Brasil já possuem sites dedicados a tornar acessíveis dados públicos, e alguns desses já estão disponíveis como Linked Data. A motivação dos governos pela disponibilização de dados abertos é justificada pela esperança do aumento do envolvimento dos cidadãos no governo, do aumento da transparência e da melhoria na tomada de decisão, alinhadas com a ambição de governos mais inteligentes (Bertot \& Choi, 2013; Conradie \& Choenni, 2014).

\section{METODOLOGIA}

Para a concepção do modelo apresentado nessa pesquisa, foram utilizadas referências coletadas sobre inovação e, mais especificamente sobre os indicadores de inovação compostos. Além disso, uma busca sistemática de literatura foi conduzida com o objetivo de identificar os modelos de indicadores utilizados para a avaliação da inovação regional.

Esta busca foi realizada no mês de dezembro de 2014 nas bases científicas Scopus e Web Of Science, utilizando como termos de busca regional innovation AND (indicator OR index). Dos 199 artigos resultantes da busca, 96 foram selecionados após a aplicação dos critérios de exclusão: a) exclusão de duplicidades; b) exclusão de artigos sem texto 
completo disponível para download gratuito; c) exclusão de artigos que não se encaixavam no contexto. A busca e seus resultados são relatados em maiores detalhes em Speroni, Macedo e Gauthier (2016b).

Os 96 artigos selecionados foram analisados, buscando identificar aqueles que utilizavam, ou propunham, algum modelo de índice de inovação, pelo agrupamento de múltiplos indicadores. Destes, 52 artigos foram selecionados, e os tipos de indicadores por eles utilizados foram catalogados, e suas características estão sendo levadas em consideração para a construção do modelo de referência aqui proposto.

Quanto à sua natureza, é de uma pesquisa aplicada (tecnológica), definida por Gil (2008) como aquela que, embora dependa das descobertas e se enriqueça com o desenvolvimento da pesquisa básica, tem como característica fundamental o interesse na aplicação, utilização e consequências práticas dos conhecimentos e no desenvolvimento de artefatos (Cupani, 2006). Tais características aplicam-se a este estudo, uma vez que o trabalho nela descrito visa a proposição de um modelo que aplica de forma prática os conceitos abordados na literatura, fornecendo um artefato para a representação de indicadores de inovação regional, suportado pelas tecnologias semânticas que embasam os dados ligados.

Quanto ao seu objetivo, caracteriza-se como uma pesquisa exploratória, aquela que tem como principal finalidade desenvolver, esclarecer e modificar conceitos e ideias, tendo em vista a formulação de problemas mais precisos ou hipóteses pesquisáveis para estudos posteriores (Gil, 2008).

Visando ao atendimento do objetivo de que o modelo seja suportado por dados ligados, fez-se necessária a representação semântica do modelo. Para tal, uma ontologia foi construída, cuja primeira versão foi desenvolvida segundo as etapas propostas no ontoKEM (Todesco, Rautenberg, Speroni, Guembarovski, \& Gauthier, 2009), que aplica conceitos e etapas de diferentes metodologias de desenvolvimento de ontologias.

\section{MODELO DE REFERÊNCIA DE INDICADORES DA INOVAÇÃO REGIONAL SUPORTADO POR DADOS LIGADOS}

A figura 1 apresenta uma representação conceitual da abordagem na qual se insere o modelo de referência proposto, considerando seu uso como base a definição de indicadores específicos usando da tecnologia de dados ligados.

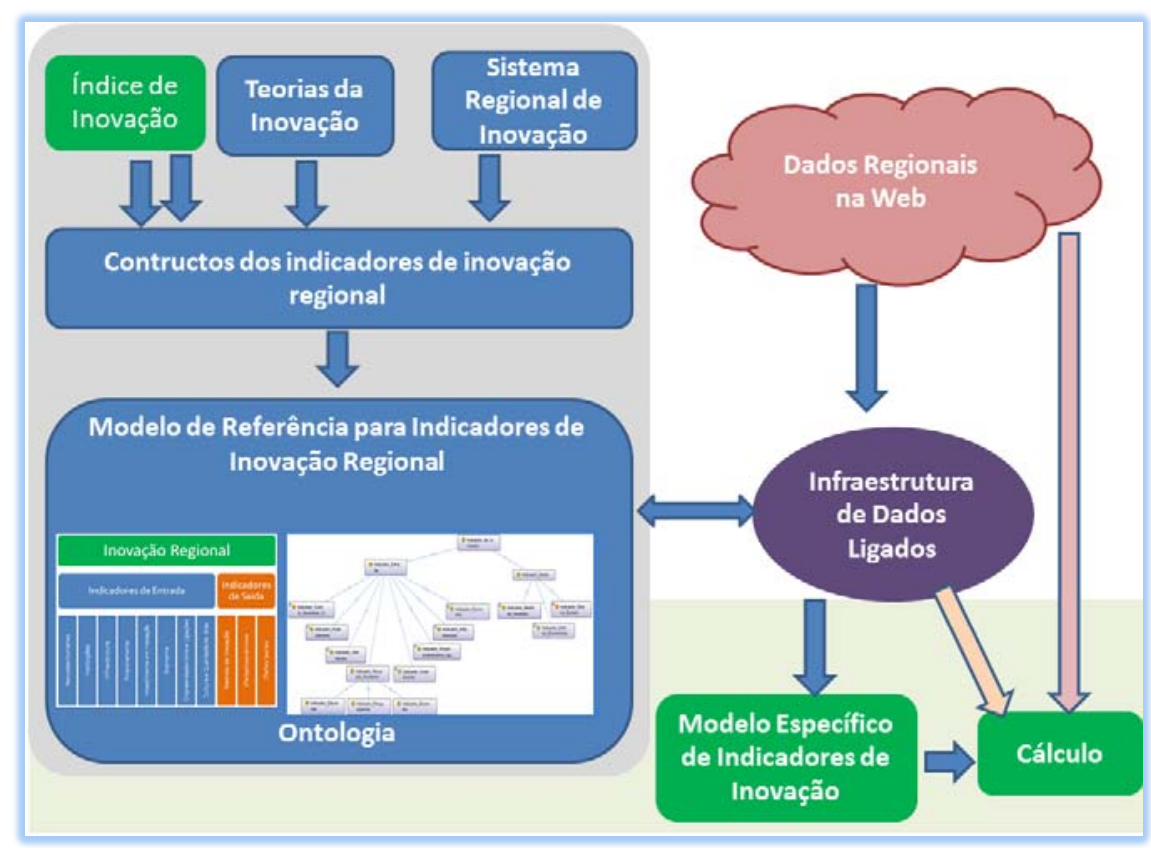

Figura 1 - Modelo de Referência para Indicadores de Inovação Regional

Fonte: Os autores (2016).

Além da representação conceitual do modelo, o mesmo é representado na forma de uma ontologia e publicado em uma infraestrutura específica, de forma que estejam acessíveis na Web e aptos para o processamento automatizado.

O cenário de utilização previsto para o modelo de referência proposto prevê que diferentes modelos indicadores compostos para a inovação regional, a exemplo dos encontrados na literatura, possam ser nele representados. Ao adotarse um modelo específico para a mensuração da inovação regional, é possível, portanto, instanciá-lo no modelo de referência. 
A instanciação de diferentes modelos específicos, juntamente com seus dados, na forma de dados ligados permite que um mesmo conjunto de dados possa vir a ser reutilizado por mais de um modelo. Além disso, no caso da opção pela definição de um novo modelo específico de indicadores para a inovação regional, o modelo ora proposto visa servir de ponto de partida, como uma referência dos constructos a serem abordados.

Uma vez que os conjuntos de dados estejam associados a um determinado modelo específico, e publicados na forma de dados ligados, os processamentos necessários para os cálculos de índices de inovação definidos no modelo podem ser efetuados.

\subsection{Modelo Conceitual de Indicadores de Inovação Regional}

Quanto à forma de concepção dos indicadores de inovação, sua estrutura e componentes, a literatura apresenta diferentes abordagens. Conforme observam Zabala Iturriagagoitia, Jiménez Saez, and Gutiérrez Gracia (2005), a maioria dos indicadores disponíveis e estatísticas de inovação consideram os sistemas de inovação como sistemas de entradasaída (input-output), com ênfase particular nos recursos empregados.

A definição de um modelo conceitual para indicadores da inovação regional, concebido pela identificação e catalogação de indicadores utilizados em modelos encontrados na literatura, é descrita por Speroni, Macedo, and Gauthier (2016a). O modelo propõe quatro níveis de classificação para os indicadores, que são formalmente descritos e exemplificados. A Figura 2 apresenta a classificação dos indicadores.

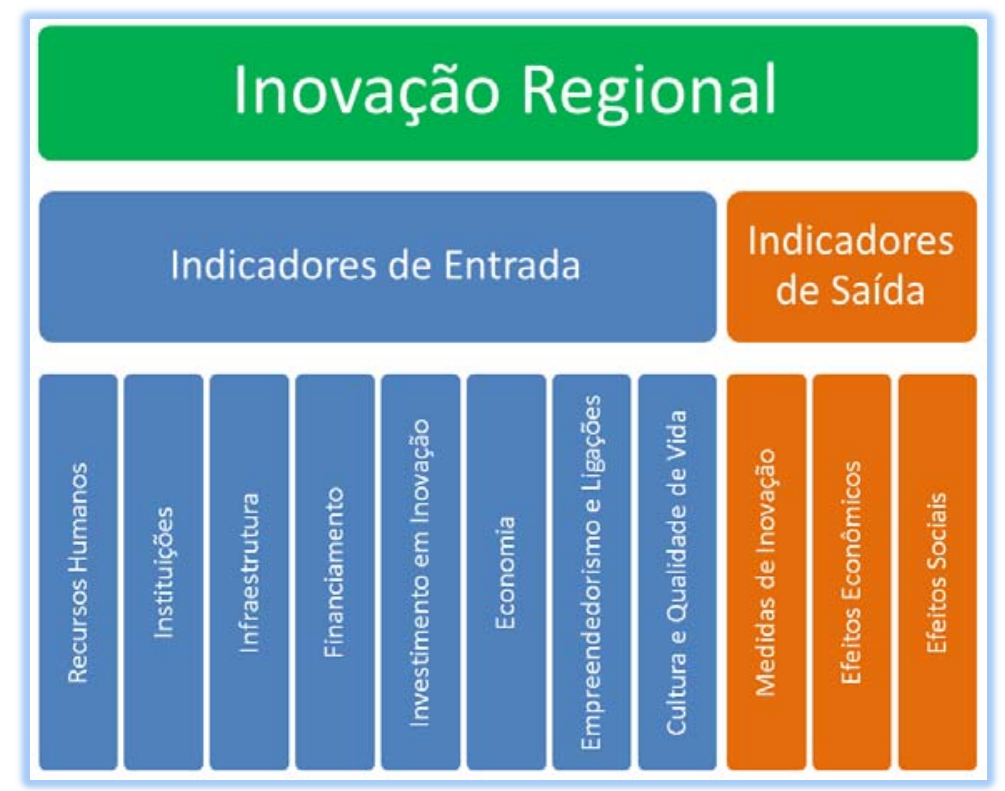

Figura 2 - Classificação dos Indicadores de Inovação Regional

Fonte: Speroni et al. (2016a).

O primeiro nível do modelo classifica os indicadores entre aqueles considerados de entrada e os de saída. Os indicadores de entrada são aqueles que representam as condições que levam um sistema de inovação a desempenhar seu papel. A seguir, as classificações dos indicadores de entrada são apresentadas no Quadro 1.

\begin{tabular}{|l|l|}
\hline \multicolumn{1}{|c|}{$\begin{array}{l}\text { Indicadores de } \\
\text { Entrada }\end{array}$} & \multicolumn{1}{c|}{ Definição } \\
\hline $\begin{array}{l}\text { Recursos } \\
\text { Humanos }\end{array}$ & $\begin{array}{l}\text { Compreende indicadores relativos à educação e a disponibilidade de força de trabalho } \\
\text { altamente qualificada e educada. }\end{array}$ \\
\hline Instituições & $\begin{array}{l}\text { Agrupa indicadores associados às condições de um sistema de inovação de prover uma boa } \\
\text { governança e níveis de proteção e incentivos essenciais à inovação. }\end{array}$ \\
\hline $\begin{array}{l}\text { Ambiente de } \\
\text { Inovação / } \\
\text { Infraestrutura }\end{array}$ & $\begin{array}{l}\text { Agrupa indicadores relativos à infraestrutura que dá suporte à inovação, tais como a } \\
\text { disponibilidade de tecnologias de informação e comunicação (TICs), a cobertura de estradas } \\
\text { pavimentadas, instituições de ensino e de institutos de pesquisa. }\end{array}$ \\
\hline $\begin{array}{l}\text { Financiamento / } \\
\text { Apoio à Inovação }\end{array}$ & $\begin{array}{l}\text { Agrupa indicadores que dizem respeito à disponibilidade de crédito, fundos de investimentos e } \\
\text { fontes de financiamento para as atividades de inovação, bem como do apoio governamental. }\end{array}$ \\
\hline $\begin{array}{l}\text { Investimento em } \\
\text { Inovação }\end{array}$ & $\begin{array}{l}\text { Compreende os indicadores que tratam da quantidade de recursos investidos nas atividades de } \\
\text { inovação. São exemplos o dispêndio público ou privado em Pesquisa e Desenvolvimento, o }\end{array}$ \\
\hline
\end{tabular}




\begin{tabular}{|l|l|}
\hline & pessoal alocado em atividades de P\&D, a compra de tecnologia. \\
\hline $\begin{array}{l}\text { Economia e } \\
\text { Mercado de } \\
\text { Trabalho }\end{array}$ & $\begin{array}{l}\text { As variáveis deste conjunto estão relacionadas às condições econômicas da região. Dentre elas, } \\
\text { citam-se indicadores como o GDP, ou o PIB, ou o nível de emprego. }\end{array}$ \\
\hline $\begin{array}{l}\text { Empreendedoris- } \\
\text { mo e Ligações }\end{array}$ & $\begin{array}{l}\text { Esta categoria engloba indicadores que representam as características da região em relação ao } \\
\text { desenvolvimento do empreendedorismo e de arranjos e da colaboração entre os diferentes } \\
\text { atores do sistema, tais como treinamentos para o empreendedorismo e as relações } \\
\text { universidade-indústria. }\end{array}$ \\
\hline $\begin{array}{l}\text { Cultura e } \\
\text { Qualidade de } \\
\text { vida }\end{array}$ & $\begin{array}{l}\text { Compreende indicadores que descrevem as características de uma região em relação ao } \\
\text { saneamento ambiental, qualidade de vida dos residentes, cultura e participação social. Exemplos } \\
\text { desse tipo são o consumo industrial de água, consumo de energia, reservas de áreas, } \\
\text { investimento em cultura, existência de teatros, ou participação em atividades voluntárias. }\end{array}$ \\
\hline
\end{tabular}

\section{Quadro 1: Indicadores de Entrada}

Fonte: Adaptado de Speroni et al. (2016a).

De forma semelhante, o quadro 2 apresenta os indicadores de saída, que representam os resultados obtidos pelo sistema de inovação.

\begin{tabular}{|l|l|}
\hline \multicolumn{1}{|c|}{$\begin{array}{c}\text { Indicadores de } \\
\text { Saída }\end{array}$} & Definição \\
\hline $\begin{array}{l}\text { Medidas de } \\
\text { Inovação }\end{array}$ & $\begin{array}{l}\text { Estão incluídos neste grupo os indicadores que dizem respeito aos resultados de } \\
\text { conhecimentos gerados pelas atividades de inovação, tais como a quantidade de empresas que } \\
\text { inovaram em produtos ou serviços, a quantidade de empresas que inovaram em marketing ou } \\
\text { em processos organizacionais, o número de patentes solicitadas e concedidas e o número de } \\
\text { publicações científicas. }\end{array}$ \\
\hline $\begin{array}{l}\text { Efeitos } \\
\text { Econômicos }\end{array}$ & $\begin{array}{l}\text { Compreende os indicadores que capturam o sucesso econômico da inovação, tais como a } \\
\text { porcentagem de vendas relativas a produtos inovadores ou a exportação de serviços } \\
\text { inovadores. }\end{array}$ \\
\hline Efeitos Sociais & $\begin{array}{l}\text { Uma vez que os resultados da inovação trazem benefícios às pessoas e ao planeta, há um } \\
\text { reconhecimento cada vez maior sobre os efeitos sociais da inovação. }\end{array}$ \\
\hline
\end{tabular}

\section{Quadro 2: Indicadores de Saída}

Fonte: Adaptado de Speroni et al. (2016a).

A próxima seção descreve os passos da construção de uma ontologia para representação do modelo de referência proposto, de forma que o mesmo possa ser mais facilmente reutilizado para a construção de modelos específicos suportados por Dados Ligados.

\subsection{Modelo de Referência Suportado por Dados Ligados}

Além de uma organização conceitual para a classificação dos indicadores de inovação regional, pretende-se que o modelo aqui proposto possibilite a publicação destes indicadores segundo os preceitos dos dados ligados. Para isto, é necessário que os conceitos explicitados sejam codificados em linguagem formal de representação de ontologias.

\subsubsection{Construção da Ontologia.}

Uma das características dos dados ligados é que os dados são autodescritivos. Conforme observam Bizer et al. (2009), se uma aplicação encontra dados que estão descritos em um vocabulário não familiar, pode consultar pelas URls que identificam os termos do vocabulário para encontrar suas definições. Assim, o modelo conceitual aqui proposto deve fornecer um vocabulário, representado na forma de uma ontologia, para descrição e os dados.

A construção de uma ontologia é um processo de modelagem, e diferentes metodologias podem ser aplicadas para este fim. Neste trabalho, está sendo utilizada como apoio para o desenvolvimento a ferramenta ontoKEM (Todesco et al., 2009). O ontoKEM utiliza conceitos oriundos de diferentes metodologias, proporcionando um ambiente Web, onde as atividades são agrupadas em quatro fases: competência, vocabulário, hierarquia de classes e dicionário de classes.

\subsubsection{Publicação e Uso da Ontologia.}


A ontologia criada tem por finalidade explicitar a representação do modelo, tornando-o um vocabulário aplicável para o mapeamento de indicadores de diferentes modelos específicos para a inovação regional. Sua codificação na linguagem RDF a torna apta a ser publicada em uma infraestrutura de dados ligados.

De forma semelhante ao que acontece com qualquer conjunto de dados ligados, quando carregada em um servidor de dados ligados, a ontologia passa a estar disponível para consultas SPARQL. Dessa forma, a representação dos recursos nela descritos está acessível também via navegador Web.

\subsection{Infraestrutura para a Publicação de Dados Estatísticos Ligados sobre a Inovação Regional}

O Modelo de Referência proposto tem por característica a exploração do potencial de utilização de dados disponíveis na Web. Pela aplicação das técnicas de dados ligados, os dados oriundos de diferentes fontes e publicadores podem ganhar nova finalidade ao serem vinculados ao modelo ora proposto.

Grande parte dos dados com potencial utilização para os indicadores de inovação são disponibilizados por órgãos públicos em função de iniciativas de transparência e legislações específicas, tais como a Lei de Acesso à Informação (Brasil, 2011). Assim, embora haja uma suposta disponibilidade de dados, eles são, tipicamente, heterogêneos e não têm um tratamento estatístico integrado, além de não haver uma forma de expor, compartilhar ou interligar os dados para que possam ser acrescentadas mais informações (Milošević, Janev, Spasić, Milojković, \& Vranes, 2012; Zancanaro, Pizzol, Speroni, Todesco, \& Gauthier, 2013; Zapilko \& Mathiak, 2011).

Considerando a característica dos dados comumente disponibilizados por tais iniciativas, predominantemente estatísticos, são aplicáveis os modelos de cubos de dados multidimensionais, característicos dos sistemas OLAP (Online Analytical Proccessing), conforme a abordagem para publicação de Dados Ligados estatísticos apresentados por Zancanaro et al. (2013).

A Figura 3 apresenta a abordagem proposta para a obtenção, transformação e publicação dos dados ligados sobre a inovação regional.

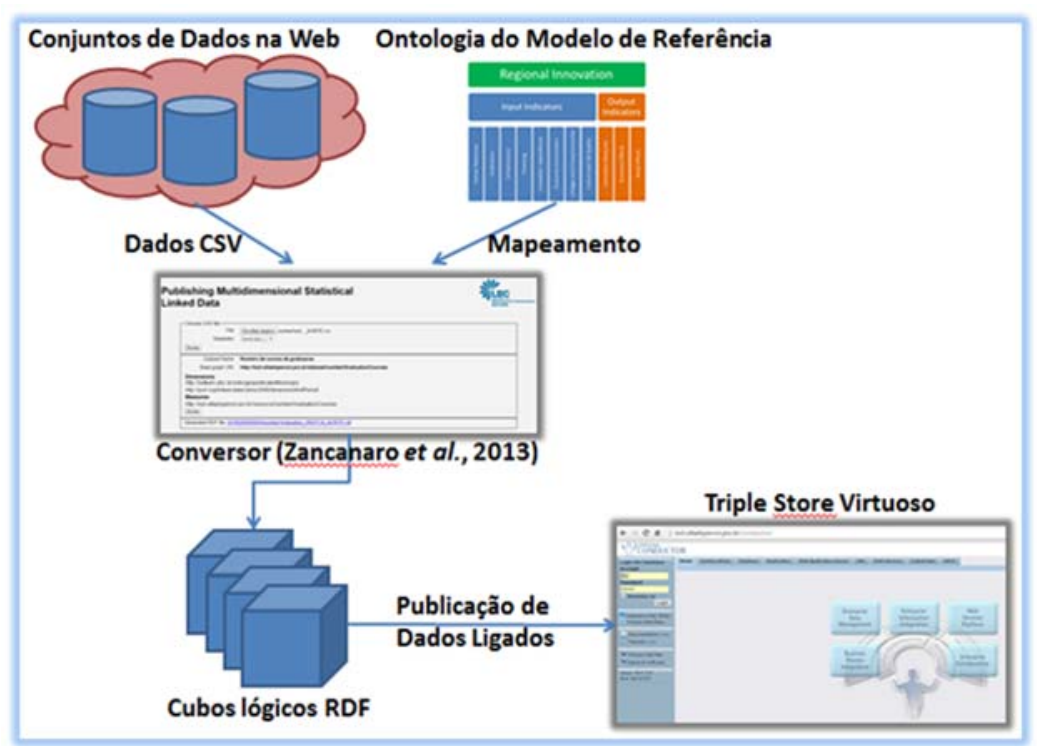

Figura 3 - Publicação de Dados Ligados sobre a Inovação Regional

Fonte: Autores (2016).

O primeiro passo consiste da identificação de fontes de dados, onde devem ser observados critérios como a precisão, temporalidade, completude e consistência dos dados. As principais fontes de dados sugeridas são as páginas de órgãos governamentais, que, por força de lei, devem publicar seus conjuntos de dados para acesso público.

Uma vez identificadas as fontes, os dados obtidos devem ser transformados, de maneira tal que sejam associados aos indicadores de inovação que representam. Neste passo, a ontologia que descreve os conceitos e interrelações propostos no modelo é utilizada como referência para o mapeamento semântico dos dados e transformação automatizada, de acordo com o processo e a ferramenta descritos por Zancanaro et al. (2013).

Os dados resultantes do processo de mapeamento e transformação são codificados no vocabulário RDF Datacube (Cyganiak, Reynolds, \& Tennison, 2010), em uma modelagem lógica de cubos multidimensionais OLAP, e podem ser carregados em um servidor de Dados Ligados do tipo Triple Store. 
A publicação dos dados em servidores do tipo Triple Store possibilita que estejam acessíveis por meio de consultas SPARQL. Além disso, pelo fato de estarem disponíveis segundo uma estrutura padronizada de cubos de Dados Estatísticos Ligados, usando o vocabulário RDF Cube, as operações de consulta também são padronizadas, possibilitando seu consumo por diferentes tipos de aplicação, em especial aquelas de caráter analítico, como as que auxiliam a tomada de decisão na gestão da inovação.

\section{CONSIDERAÇÕES FINAIS}

A inovação regional costuma ser analisada na literatura como um processo sistêmico, que acontece pela interação entre diferentes tipos de atores e é diretamente influenciada pelas pré-condições do ambiente que os cerca. Dessa forma, a mensuração da inovação regional deve levar em conta os múltiplos aspectos desse Sistema Regional de Inovação, e necessita de indicadores que os descrevam.

De forma semelhante ao que acontece com os Sistemas Nacionais de Inovação, a abordagem comumente adotada para a mensuração da inovação regional envolve a adoção de modelos de indicadores compostos, cujas escolhas de variáveis e formas de organização variam entre os autores. Este estudo apresentou a concepção de um modelo de referência baseado em uma busca de literatura, onde foram identificadas 52 publicações, que foram catalogadas.

A partir dos dados levantados, foram identificados os indicadores mais utilizados pelos autores e, de acordo com suas características, foram agrupados em classes que representam os constructos que estão relacionados com a inovação regional. As classes foram organizadas em um modelo conceitual com estrutura hierárquica composta por três níveis.

O modelo conceitual foi representado na forma de uma ontologia, onde os conceitos representados foram descritos e interligados para que, posteriormente, fosse publicada na forma de uma infraestrutura específica para dados ligados. A ontologia publicada neste formato torna-se acessível tanto via navegador Web, quanto via interface de consulta SPARQL, possibilitando seu consumo por aplicações computacionais.

Por suas características, os dados ligados da ontologia podem ser facilmente consultados como fonte de referência para a definição de modelos específicos de indicadores da inovação regional. Os indicadores catalogados e ligados ao modelo de referência podem servir como um ponto de partida para a escolha de indicadores a serem utilizados.

As sugestões de pesquisa apresentadas possibilitam que, além do desenvolvimento de produtos para casos específicos, sejam criadas as condições para o aprendizado e difusão das tecnologias semânticas. Assim, o envolvimento de acadêmicos e de profissionais de órgãos públicos ou de empresas privadas, que tenham algum tipo de participação na produção, publicação ou consumo de dados, representará um importante passo na direção de difundir a publicação dos dados ligados.

\section{REFERÊNCIAS}

Adams, R., Bessant, J., \& Phelps, R. (2006). Innovation management measurement: A review. International Journal of Management Reviews, 8(1), 27.

Berners-Lee, T. (2006). Linked Data: Design Issues. Recuperado de: http://www.w3.org/Designlssues/LinkedData.html

Bertot, J. C., \& Choi, H. (2013). Big Data and e-Government: Issues, Policies, and Recommendations. Paper presented at the 14th Annual Internatinal Conference on Digital Government Research, Quebec, Canada.

Bizer, C., Heath, T., \& Berners-Lee, T. (2009). Linked data - The story so far. International Journal on Semantic Web and Information Systems, 5(3), 22.

Bradley, F. (2009). Discovering Linked Data. Library Journal, 134(7), 3.

Brasil. (2011). Lei No 12.527, de 18 de Novembro de 2011. Regula o acesso a informações previsto no inciso XXXIII do art. 5o, no inciso II do § 30 do art. 37 e no $§ 20$ do art. 216 da Constituição Federal; altera a Lei no 8.112, de 11 de dezembro de 1990; revoga a Lei no 11.111, de 5 de maio de 2005, e dispositivos da Lei no 8.159, de 8 de janeiro de 1991; e dá outras providências.

Conradie, P., \& Choenni, S. (2014). On the barriers for local government releasing open data. Government Information Quarterly, 31. doi: 10.1016/j.giq.2014.01.003

Cornell University, INSEAD, \& WIPO. (2013). The Global Innovation Index 2013: The local dynamics of innovation. Geneva, Switzerland: WIPO.

Crossan, M. M., \& Apaydin, M. (2010). A multi-dimensional framework of organizational innovation: A systematic review of the literature. Journal of Management Studies, 47(6), 1154-1191. doi: 10.1111/j.1467-6486.2009.00880.x

Cupani, A. (2006). La peculiaridad del conocimiento tecnológico. ScientiaeStudia, 4(3), São Paulo, p. 353-371.

Cyganiak, R., Reynolds, D., \& Tennison, J. (Producer). (2010, Novembro, 2015). The RDF Data Cube vocabulary. Retrieved from http://publishing-statistical-data.googlecode.com/svn/trunk/specs/src/main/html/cube.html 
Doloreaux, D., \& Parto, S. (2004). Regional innovation systems: A critical review. Paper presented at the XLème Colloque de L'ASRDLF, Bruxelles.

European Union. (2014). Innovation Union Scoreboard 2014. Brussels: European Comission.

Gil, A. C. (2008). Métodos e técnicas de pesquisa social (6 ed.). Sao Paulo: Editora Atlas.

Heath, T. (2011). Linked Data - Welcome to the Data Network. IEEE Internet Computing, 15(6), 4.

Hyland, B., \& Wood, D. (2011). The joy of data - A cookbook for publishing linked government data on the web. In Wood, D. (Ed.). Linking government data. Fredericksburg, VA, USA: Springer.

Milošević, U., Janev, V., Spasić, M., Milojković, J., \& Vranes, S. (2012). Publishing statistical data as linked open data. Paper presented at the 2nd International Conference on Information Society, Serbia.

National Inovation Council. (2010). Introduction. Recuperado de: http://www.innovationcouncil.gov.in/index.php?option=com_content\&view=article\&id=26\&ltemid=5

OECD. (2005). Oslo Manual: Guidelines for collecting and interpreting innovation data (3 ed.). Paris, France: OECD.

OECD. (2015). Frascati Manual: Guidelines for collecting and reporting data on research and experimental development the measurement of scientific, technological and innovation activities. Paris, France: OECD.

Open Definition. (2009). Open Definition - version 1.1. Recuperado de: http://opendefinition.org/od/

Schumpeter, J. A. (2012). The theory of economic development: An inquiry into profits, capital, credit, interest, and the business cycle (16 ed.). New Jersey, USA: Transaction Publishers.

Speroni, R. D. M., Macedo, M., \& Gauthier, F. A. O. (2016a). Modelo de referência para indicadores de inovação regional. Espacios, 37(10).

Speroni, R. d. M., Macedo, M., \& Gauthier, F. A. O. (2016b). Revisão sistemática de literatura sobre indicadores da inovação regional. Espacios, 37(05).

Todesco, J. L., Rautenberg, S., Speroni, R. d. M., Guembarovski, R. H., \& Gauthier, F. Á. O. (2009). ontoKEM: A web tool for ontologies' construction and documentation. Paper presented at the International Conference on Information \& Knowledge Engineering, 2009; IKE 09, Las Vegas.

Zabala Iturriagagoitia, J. M., Jiménez Saez, F., \& Gutiérrez Gracia, A. (2005). Analysis and measurement of interactions in regional innovation systems: Need to define new network indicators. Paper presented at the 15th International Conference on Engineering Design, ICED 05, Melbourne, VIC.

Zancanaro, A., Pizzol, L. D., Speroni, R. D. M., Todesco, J. L., \& Gauthier, F. A. O. (2013). Publishing multidimensional statistical linked data. Paper presented at the eKNOW 2013, The Fifth International Conference on Information, Process, and Knowledge Management, Nice, France.

Zapilko, B., \& Mathiak, B. (2011). Performing statistical methods on linked data. Paper presented at the International Conference on Dublin Core and Metadata Applications, The Hague, Netherlands. 